\title{
Three-Point Boundary Value Problems for the Langevin Equation with the Hilfer Fractional Derivative
}

\author{
Athasit Wongcharoen, ${ }^{1}$ Bashir Ahmad, ${ }^{2}$ Sotiris K. Ntouyas $\mathbb{D}^{3,2}$ and Jessada Tariboon $\mathbb{D}^{4}$ \\ ${ }^{1}$ Department of Mechanical Engineering Technology, College of Industrial Engineering Technology, King Mongkut's University of \\ Technology North Bangkok, Bangkok 10800, Thailand \\ ${ }^{2}$ Nonlinear Analysis and Applied Mathematics (NAAM)-Research Group, Department of Mathematics, Faculty of Science, \\ King Abdulaziz University, P.O. Box 80203, Jeddah 21589, Saudi Arabia \\ ${ }^{3}$ Department of Mathematics, University of Ioannina, 45110 Ioannina, Greece \\ ${ }^{4}$ Intelligent and Nonlinear Dynamic Innovations Research Center, Department of Mathematics, Faculty of Applied Science, \\ King Mongkut's University of Technology North Bangkok, Bangkok 10800, Thailand
}

Correspondence should be addressed to Jessada Tariboon; jessada.t@sci.kmutnb.ac.th

Received 6 February 2020; Accepted 7 April 2020; Published 4 May 2020

Academic Editor: Jorge E. Macias-Diaz

Copyright (c) 2020 Athasit Wongcharoen et al. This is an open access article distributed under the Creative Commons Attribution License, which permits unrestricted use, distribution, and reproduction in any medium, provided the original work is properly cited.

\begin{abstract}
We discuss the existence and uniqueness of solutions for the Langevin fractional differential equation and its inclusion counterpart involving the Hilfer fractional derivatives, supplemented with three-point boundary conditions by means of standard tools of the fixed-point theorems for single and multivalued functions. We make use of Banach's fixed-point theorem to obtain the uniqueness result, while the nonlinear alternative of the Leray-Schauder type and Krasnoselskii's fixed-point theorem are applied to obtain the existence results for the single-valued problem. Existence results for the convex and nonconvex valued cases of the inclusion problem are derived via the nonlinear alternative for Kakutani's maps and Covitz and Nadler's fixed-point theorem respectively. Examples illustrating the obtained results are also constructed. (2010) Mathematics Subject Classifications. This study is classified under the following classification codes: 26A33; 34A08; 34A60; and 34B15.
\end{abstract}

\section{Introduction}

Fractional calculus is an emerging field in applied mathematics that deals with derivatives and integrals of arbitrary orders. For details and applications, we refer the reader to the texts in [1-6]. In the literature, there exist several definitions of fractional integrals and derivatives, from the most popular Riemann-Liouville and Caputo-type fractional derivatives to others such as the Hadamard fractional derivative and the Erdeyl-Kober fractional derivative. A generalization of both the Riemann-Liouville and Caputo derivatives was given by Hilfer in [7], which is known as the Hilfer fractional derivative $D^{\alpha, \beta} x(t)$ of order $\alpha$ and type $\beta \in[0,1]$. One can observe that the Hilfer fractional derivative interpolates between the Riemann-Liouville and Caputo derivatives as it reduces to the Riemann-Liouville and Caputo fractional derivatives for $\beta=0$ and $\beta=1$, respectively. Some properties and applications of the Hilfer derivative can be found in $[8,9]$ and references cited therein.

One of the important equations governing several phenomena occurring in physical sciences and electrical engineering is the Langevin differential equation, first formulated by Langevin in 1908 [10]. In recent years, several fractional variants of the Langevin equation have been introduced and studied; see, for example, [11-19] and the references cited therein.

Initial value problems involving the Hilfer fractional derivatives were studied by several authors; see for example [20-22]. Nonlocal boundary value problems for the Hilfer fractional differential equation have been discussed in [23]. In [24], the authors proved some results for initial value problems of the Langevin equation with the Hilfer fractional derivative. 
Exploring the literature on fractional order boundary value problems, we find that there does not exist any work on boundary value problems of the Langevin equation with the Hilfer fractional derivative. Motivated by this observation, we fill this gap by introducing a new class of boundary value problems of the Hilfer-type Langevin fractional differential equation with three-point nonlocal boundary conditions. In precise terms, we investigate the existence and uniqueness criteria for the solutions of the following nonlocal boundary value problem:

$$
\begin{gathered}
{ }^{H} D^{\alpha_{1}, \beta_{1}}\left({ }^{H} D^{\alpha_{2}, \beta_{2}}+\lambda\right) x(t)=f(t, x(t)), \quad t \in[a, b], \\
x(a)=0 \\
x(b)=\theta x(\eta), \\
\theta \in \mathbb{R}, \eta \in(a, b),
\end{gathered}
$$

where ${ }^{H} D^{\alpha_{i}, \beta_{i}}, i=1,2$ is the Hilfer fractional derivative of order $\alpha_{i}, 0<\alpha_{i}<1$ and parameter $\beta_{i}, 0 \leq \beta_{i} \leq 1, i=1,2, \lambda \in$ $\mathbb{R}, a \geq 0$, and $f:[a, b] \times \mathbb{R} \rightarrow \mathbb{R}$ is a continuous function.

In order to study problem (1)-(2), we convert it into an equivalent fixed-point problem and then use Banach's fixed-point theorem to prove the uniqueness of its solutions. We also obtain two existence results for problem (1)-(2) by applying the nonlinear alternative of the Leray-Schauder type [25] and Krasnoselskii's fixed-point theorem [26].

As a second problem, we switch onto the multivalued analogue of (1) and (2) by considering the inclusion problem:

$$
\begin{gathered}
{ }^{H} D^{\alpha_{1}, \beta_{1}}\left({ }^{H} D^{\alpha_{2}, \beta_{2}}+\lambda\right) x(t) \in F(t, x(t)), \quad t \in[a, b], \\
x(a)=0 \\
x(b)=\theta x(\eta), \\
\theta \in \mathbb{R}, \eta \in[a, b]
\end{gathered}
$$

where $F:[a, b] \times \mathbb{R} \longrightarrow \mathscr{P}(\mathbb{R})$ is a multivalued map $(\mathscr{P}(\mathbb{R})$ is the family of all nonempty subjects of $\mathbb{R}$ ).

Existence results for problem (3)-(4) with convex and nonconvex valued maps are respectively derived by applying the nonlinear alternative for Kakutani's maps and Covitz and Nadler's fixed-point theorem for contractive maps.

The rest of the paper is organized as follows: Section 3 contains the main results for problem (1)-(2), while the existence results for problem (3)-(4) are presented in Section 4 . We recall the related background material in Section 2.

\section{Preliminaries}

In this section, we introduce some notations and definitions of fractional calculus and multivalued analysis and present preliminary results needed in our proofs later [1].
Definition 1. The Riemann-Liouville fractional integral of order $\alpha>0$ for a continuous function $u:[a, \infty) \longrightarrow \mathbb{R}$ is defined by

$$
I^{\alpha} u(t)=\frac{1}{\Gamma(\alpha)} \int_{a}^{t}(t-s)^{\alpha-1} u(s) d s,
$$

provided that the right-hand side exists on $(a, \infty)$.

Definition 2. The Riemann-Liouville fractional derivative of order $\alpha>0$ of a continuous function $u$ is defined by

$$
\begin{array}{r}
{ }^{R L} D^{\alpha} u(t):=D^{n} I^{n-\alpha} u(t)=\frac{1}{\Gamma(n-\alpha)}\left(\frac{d}{d t}\right)^{n} \int_{a}^{t}(t-s)^{n-\alpha-1} u(s) d s, \\
n-1<\alpha<n,
\end{array}
$$

where $n=[\alpha]+1,[\alpha]$ denotes the integer part of real number $\alpha$, provided that the right-hand side is point-wise defined on $(a, \infty)$.

Definition 3. The Caputo fractional derivative of order $\alpha>0$ of a continuous function $u$ is defined by

$$
\begin{array}{r}
{ }^{C} D^{\alpha} u(t):=I^{n-\alpha} D^{n} u(t)=\frac{1}{\Gamma(n-\alpha)} \int_{a}^{t}(t-s)^{n-\alpha-1}\left(\frac{d}{d s}\right)^{n} u(s) d s, \\
n-1<\alpha<n,
\end{array}
$$

provided that the right-hand side is point-wise defined on $(a, \infty)$.

Definition 4 (Hilfer fractional derivative $[7,8]$ ). The Hilfer fractional derivative of order $\alpha$ and parameter $\beta$ of a function (also known as the generalized Riemann-Liouville fractional derivative) is defined by

$$
{ }^{H} D^{\alpha, \beta} u(t)=I^{\beta(n-\alpha)} D^{n} I^{(1-\beta)(n-\alpha)} u(t),
$$

where $n-1<\alpha<n, 0 \leq \beta \leq 1, t>a$, and $D=(d / d t)$.

Remark 5. When $\beta=0$, the Hilfer fractional derivative corresponds to the Riemann-Liouville fractional derivative:

$$
{ }^{H} D^{\alpha, 0} u(t)=D^{n} I^{n-\alpha} u(t)
$$

while $\beta=1$ in the definition of the Hilfer fractional derivative corresponds to the Caputo fractional derivative:

$$
{ }^{H} D^{\alpha, 1} u(t)=I^{n-\alpha} D^{n} u(t)
$$

In the following lemma, we present the compositional property of the Riemann-Liouville fractional integral operator with the Hilfer fractional derivative operator. 
Lemma 6 (see [8]). Let $f \in L(a, b), n-1<\alpha \leq n, n \in \mathbb{N}, 0 \leq$ $\beta \leq 1$, and $I^{(n-\alpha)(1-\beta)} f \in A C^{k}[a, b]$. Then

$$
\begin{aligned}
\left(I^{\alpha H} D^{\alpha, \beta} f\right)(t)= & f(t)-\sum_{k=0}^{n-1} \frac{(t-a)^{k-(n-\alpha)(1-\beta)}}{\Gamma(k-(n-\alpha)(1-\beta)+1)} \\
& \cdot \lim _{t \rightarrow a^{+}} \frac{d^{k}}{d t^{k}}\left(I^{(1-\beta)(n-\alpha)} f\right)(t) .
\end{aligned}
$$

The following lemma deals with a linear variant of boundary value problem (1)-(2).

Lemma 7. Let $a \geq 0,0<\alpha_{i}<1, \quad \gamma_{i}=\alpha_{i}+\beta_{i}-\alpha_{i} \beta_{i}, \quad i=1,2$, $\gamma_{1}+\alpha_{2}>1$, and $h \in C([a, b], \mathbb{R})$. Then, the function $x$ is a solution of the boundary value problem:

$$
\begin{gathered}
{ }^{H} D^{\alpha_{1}, \beta_{1}}\left({ }^{H} D^{\alpha_{2}, \beta_{2}}+\lambda\right) x(t)=h(t), \\
x(a)=0 \\
x(b)=\theta x(\eta)
\end{gathered}
$$

if and only if

$$
\begin{aligned}
x(t)= & I^{\alpha_{1}+\alpha_{2}} h(t)-\lambda I^{\alpha_{2}} x(t)+\frac{(t-a)^{\gamma_{1}+\alpha_{2}-1}}{\Lambda \Gamma\left(\gamma_{1}+\alpha_{2}\right)} \\
& \cdot\left[\theta I^{\alpha_{1}+\alpha_{2}} h(\eta)-\lambda \theta I^{\alpha_{2}} x(\eta)-I^{\alpha_{1}+\alpha_{2}} h(b)+\lambda I^{\alpha_{2}} x(b)\right],
\end{aligned}
$$

where it is assumed that

$$
\Lambda=\frac{1}{\Gamma\left(\gamma_{1}+\alpha_{2}\right)}\left[(b-a)^{\gamma_{1}+\alpha_{2}-1}-\theta(\eta-a)^{\gamma_{1}+\alpha_{2}-1}\right] \neq 0 .
$$

Proof. Applying the Riemann-Liouville fractional integral of order $\alpha_{1}$ to both sides of (12), we obtain by using Lemma 6

$$
{ }^{H} D^{\alpha_{2}, \beta_{2}} x(t)+\lambda x(t)=\frac{c_{0}}{\Gamma\left(\gamma_{1}\right)}(t-a)^{\gamma_{1}-1}+I^{\alpha_{1}} h(t),
$$

where $c_{0}$ is an arbitrary constant and $\left(2-\alpha_{1}\right)\left(1-\beta_{1}\right)=$ $2-\gamma_{1}$. Applying the Riemann-Liouville fractional integral of order $\alpha_{2}$ to both sides of (16), we obtain

$$
\begin{aligned}
I^{\alpha_{2} H} D^{\alpha_{2}, \beta_{2}} x(t) & =I^{\alpha_{1}+\alpha_{2}} h(t)-\lambda I^{\alpha_{2}} x(t)+\frac{c_{0}}{\Gamma\left(\gamma_{1}\right)} I^{\alpha_{2}}(t-a)^{\gamma_{1}-1} \\
& =I^{\alpha_{1}+\alpha_{2}} h(t)-\lambda I^{\alpha_{2}} x(t)+\frac{c_{0}}{\Gamma\left(\gamma_{1}+\alpha_{2}\right)}(t-a)^{\gamma_{1}+\alpha_{2}-1} .
\end{aligned}
$$

Applying Lemma 6 to (17), we obtain

$$
\begin{aligned}
x(t)= & I^{\alpha_{1}+\alpha_{2}} h(t)-\lambda I^{\alpha_{2}} x(t)+\frac{c_{0}}{\Gamma\left(\gamma_{1}+\alpha_{2}\right)}(t-a)^{\gamma_{1}+\alpha_{2}-1} \\
& +\frac{c_{1}}{\Gamma\left(\gamma_{2}\right)}(t-a)^{\gamma_{2}-1} .
\end{aligned}
$$

Using $x(a)=0$ in (18), we obtain $c_{1}=0$, and hence we get

$$
x(t)=I^{\alpha_{1}+\alpha_{2}} h(t)-\lambda I^{\alpha_{2}} x(t)+\frac{c_{0}}{\Gamma\left(\gamma_{1}+\alpha_{2}\right)}(t-a)^{\gamma_{1}+\alpha_{2}-1} .
$$

Next, combining the condition $x(b)=\theta x(\eta)$ with (19), we have

$$
c_{0}=\frac{1}{\Lambda}\left[\theta I^{\alpha_{1}+\alpha_{2}} h(\eta)-\lambda \theta I^{\alpha_{2}} x(\eta)-I^{\alpha_{1}+\alpha_{2}} h(b)+\lambda I^{\alpha_{2}} x(b)\right] .
$$

Substituting the value of $c_{0}$ in (19) yields the solution (14). The converse follows by direct computation. This completes the proof.

\section{Existence and Uniqueness Results for Single- Valued Problem (1)-(2)}

In view of Lemma 7 , we define an operator $\mathscr{A}: \mathscr{C} \longrightarrow \mathscr{C}$ associated with problem (1)-(2) by

$$
\begin{aligned}
(\mathscr{A} x)(t)= & I^{\alpha_{1}+\alpha_{2}} f(t, x(t))-\lambda I^{\alpha_{2}} x(t)+\frac{(t-a)^{\gamma_{1}+\alpha_{2}-1}}{\Lambda \Gamma\left(\gamma_{1}+\alpha_{2}\right)} \\
& \cdot\left[\theta I^{\alpha_{1}+\alpha_{2}} f(\eta, x(\eta))-\lambda \theta I^{\alpha_{2}} x(\eta)-I^{\alpha_{1}+\alpha_{2}} f(b, x(b))\right. \\
& \left.+\lambda I^{\alpha_{2}} x(b)\right],
\end{aligned}
$$

where $\mathscr{C}=C([a, b], \mathbb{R})$ denotes the Banach space of all continuous functions from $[a, b]$ into $\mathbb{R}$ with the norm $\|x\|:=$ $\sup \{|x(t)|: t \in[a, b]\}$. One can observe that the existence of a fixed point of operator $\mathscr{A}$ implies the existence of a solution for problem (1)-(2).

For computational convenience, we introduce the following notations:

$$
\begin{array}{r}
\Omega_{1}=\frac{(b-a)^{\alpha_{1}+\alpha_{2}}}{\Gamma\left(\alpha_{1}+\alpha_{2}+1\right)}+\frac{(b-a)^{\gamma_{1}+\alpha_{2}-1}}{|\Lambda| \Gamma\left(\gamma_{1}+\alpha_{2}\right)} \\
\times\left[\frac{(b-a)^{\alpha_{1}+\alpha_{2}}}{\Gamma\left(\alpha_{1}+\alpha_{2}+1\right)}+|\theta| \frac{(\eta-a)^{\alpha_{1}+\alpha_{2}}}{\Gamma\left(\alpha_{1}+\alpha_{2}+1\right)}\right], \\
\Omega_{2}=|\lambda|\left\{\frac{(b-a)^{\alpha_{2}}}{\Gamma\left(\alpha_{2}+1\right)}+\frac{(b-a)^{\gamma_{1}+\alpha_{2}-1}}{|\Lambda| \Gamma\left(\gamma_{1}+\alpha_{2}\right)}\right. \\
\left.\times\left[\frac{(b-a)^{\alpha_{2}}}{\Gamma\left(\alpha_{2}+1\right)}+|\theta| \frac{(\eta-a)^{\alpha_{2}}}{\Gamma\left(\alpha_{2}+1\right)}\right]\right\} .
\end{array}
$$


Now, we present our main results for boundary value problem (1)-(2). Our first existence result is based on the well-known Krasnoselskii's fixed-point theorem [26].

Theorem 8. Assume that the following conditions hold:

$\left(H_{1}\right) f:[a, b] \times \mathbb{R} \longrightarrow \mathbb{R}$ is a continuous function such that $|f(t, x)| \leq \varphi(t), \forall(t, x) \in[a, b] \times \mathbb{R}$, with $\varphi \in C([a$, $\left.b], \mathbb{R}^{+}\right)$

$\left(H_{2}\right) \Omega_{2}<1$, where $\Omega_{2}$ is given by (23)

Then, there exists at least one solution for problems (1) and (2) on $[a, b]$.

Proof. In order to verify the hypothesis of Krasnoselskii's fixed-point theorem [26], we split operator $\mathscr{A}$ defined by (21) into the sum of two operators $\mathscr{A}_{1}$ and $\mathscr{A}_{2}$ on the closed ball $B_{\rho}=\{x \in \mathscr{C}:\|x\| \leq \rho\}$ with $\rho \geq\left(\left(\|\varphi\| \Omega_{1}\right) /\left(1-\Omega_{2}\right)\right)$, $\sup _{t \in[a, b]} \varphi(t)=\|\varphi\|$, where

$$
\begin{aligned}
\mathscr{A}_{1} x(t)= & I^{\alpha_{1}+\alpha_{2}} f(t, x(t))+\frac{(t-a)^{\gamma_{1}+\alpha_{2}-1}}{\Lambda \Gamma\left(\gamma_{1}+\alpha_{2}\right)} \\
& \cdot\left[\theta I^{\alpha_{1}+\alpha_{2}} f(\eta, x(\eta))-I^{\alpha_{1}+\alpha_{2}} f(b, x(b))\right], \quad t \in[a, b],
\end{aligned}
$$

and

$$
\begin{array}{r}
\mathscr{A}_{2} x(t)=-\lambda I^{\alpha_{2}} x(t)+\frac{(t-a)^{\gamma_{1}+\alpha_{2}-1}}{\Lambda \Gamma\left(\gamma_{1}+\alpha_{2}\right)}\left[-\lambda \theta I^{\alpha_{2}} x(\eta)+\lambda I^{\alpha_{2}} x(b)\right], \\
t \in[a, b] .
\end{array}
$$

For any $x, y \in B_{\rho}$, we have

$$
\begin{aligned}
& \left|\left(\mathscr{A}_{1} x\right)(t)+\left(\mathscr{A}_{2} y\right)(t)\right| \leq \sup _{t \in[a, b]}\left\{I^{\alpha_{1}+\alpha_{2}}|f(t, x(t))|+|\lambda| I^{\alpha_{2}}|x(t)|\right. \\
& \quad+\frac{(t-a)^{\gamma_{1}+\alpha_{2}-1}}{|\Lambda| \Gamma\left(\gamma_{1}+\alpha_{2}\right)}\left[|\theta| I^{\alpha_{1}+\alpha_{2}}|f(\eta, x(\eta))|\right. \\
& \quad+|\lambda||\theta| I^{\alpha_{2}}\left|x(\eta)+I^{\alpha_{1}+\alpha_{2}}\right| f(b, x(b)) \mid \\
& \left.\left.\quad+|\lambda| I^{\alpha_{2}}|x(b)|\right]\right\} \leq\|\varphi\| \Omega_{1}+\rho \Omega_{2} \leq \rho .
\end{aligned}
$$

This shows that $\mathscr{A}_{1} x+\mathscr{A}_{2} y \in B_{\rho}$. By using $\left(\mathrm{H}_{2}\right)$, it is easy to establish that $\mathscr{A}_{2}$ is a contraction mapping.

Continuity of operator $\mathscr{A}_{1}$ follows from that of $f$. Also, $\mathscr{A}_{1}$ is uniformly bounded on $B_{\rho}$ as

$$
\left\|\mathscr{A}_{1} x\right\| \leq \Omega_{1}\|\varphi\|
$$

Now, we prove that operator $\mathscr{A}_{1}$ is compact. Setting $\sup _{(t, x) \in[a, b] \times B_{\rho}}|f(t, x)|=\bar{f}<\infty$, we obtain

$$
\begin{aligned}
& \left|\left(\mathscr{A}_{1} x\right)\left(t_{2}\right)-\left(\mathscr{A}_{1} x\right)\left(t_{1}\right)\right| \\
& \quad=\frac{1}{\Gamma\left(\alpha_{1}+\alpha_{2}\right)} \mid \int_{a}^{t_{1}}\left[\left(t_{2}-s\right)^{\alpha_{1}+\alpha_{2}-1}-\left(t_{1}-s\right)^{\alpha_{1}+\alpha_{2}-1}\right] f(s, x(s)) d s \\
& \quad+\int_{t_{1}}^{t_{2}}\left(t_{2}-s\right)^{\alpha_{1}+\alpha_{2}-1} f(s, x(s)) d s \mid \\
& \quad+\frac{\left(t_{2}-a\right)^{\gamma_{1}+\alpha_{2}-1}-\left(t_{1}-a\right)^{\gamma_{1}+\alpha_{2}-1}}{|\Lambda| \Gamma\left(\gamma_{1}+\alpha_{2}\right)} \\
& \quad \cdot\left[|\theta| \bar{f} \frac{(\eta-a)^{\alpha_{1}+\alpha_{2}}}{\Gamma\left(\alpha_{1}+\alpha_{2}+1\right)}+\bar{f} \frac{(b-a)^{\alpha_{1}+\alpha_{2}}}{\Gamma\left(\alpha_{1}+\alpha_{2}+1\right)}\right] \\
& \quad \leq \frac{\bar{f}}{\Gamma\left(\alpha_{1}+\alpha_{2}+1\right)}\left[2\left(t_{2}-t_{1}\right)^{\alpha_{1}+\alpha_{2}}\right. \\
& \left.\quad+\left|\left(t_{2}-a\right)^{\alpha_{1}+\alpha_{2}}-\left(t_{1}-a\right)^{\alpha_{1}+\alpha_{2}}\right|\right] \\
& \quad+\frac{\left(t_{2}-a\right)^{\gamma_{1}+\alpha_{2}-1}-\left(t_{1}-a\right)^{\gamma_{1}+\alpha_{2}-1}}{|\Lambda| \Gamma\left(\gamma_{1}+\alpha_{2}\right)} \\
& \quad \cdot\left[|\theta| \bar{f} \frac{(\eta-a)^{\alpha_{1}+\alpha_{2}}}{\Gamma\left(\alpha_{1}+\alpha_{2}+1\right)}+\bar{f} \frac{(b-a)^{\alpha_{1}+\alpha_{2}}}{\Gamma\left(\alpha_{1}+\alpha_{2}+1\right)}\right] \longrightarrow 0 \text { as } t_{2}-t_{1} \longrightarrow 0,
\end{aligned}
$$

independently of $x \in B_{\rho}$. Thus, $\mathscr{A}_{1}$ is equicontinuous and hence $\mathscr{A}_{1}$ is relatively compact on $B_{\rho}$. In consequence, it follows from the Arzelá-Ascoli theorem that $\mathscr{A}_{1}$ is compact on $B_{\rho}$. In view of the foregoing arguments, we deduce that the hypothesis of Krasnoselskii's fixed-point theorem [26] holds true and consequently its conclusion implies that problem (1)-(2) has at least one solution on $[a, b]$.

Example 9. Consider the three-point boundary value problem of the Langevin equation with the Hilfer fractional derivative of the form:

$$
\left\{\begin{array}{l}
{ }^{H} D^{(4 / 5),(3 / 4)}\left({ }^{H} D^{(3 / 5),(1 / 2)}+\frac{1}{4}\right) x(t)=\frac{3 \sin ^{2} t}{5+3 t}\left(\frac{|x(t)|}{1+|x(t)|}\right)+\frac{1}{4}, \quad t \in\left[\frac{1}{3}, \frac{5}{3}\right], \\
x\left(\frac{1}{3}\right)=0, \quad x\left(\frac{5}{3}\right)=\frac{2}{7} x\left(\frac{2}{3}\right) .
\end{array}\right.
$$

Here, $\alpha_{1}=4 / 5, \alpha_{2}=3 / 5, \beta_{1}=3 / 4, \beta_{2}=1 / 2, \lambda=1 / 4, a=1$ $13, b=5 / 3, \eta=2 / 3$, and $\theta=2 / 7$. Using the given values, it is found that $\gamma_{1}=19 / 20, \gamma_{2}=4 / 5, \Lambda \approx 1.142227093, \Omega_{1} \approx$ 2.650804917 , and $\Omega_{2} \approx 0.7638622147<1$. Notice that

$$
|f(t, x)| \leq \frac{3 \sin ^{2} t}{5+3 t}+\frac{1}{4}:=\varphi(t) .
$$

Clearly the hypothesis of Theorem 8 holds true and consequently its conclusion implies that the boundary value problem (29) has at least one solution on $[1 / 3,5 / 3]$.

The Leray-Schauder Nonlinear Alternative [25] is used for our next existence result.

Theorem 10. Suppose that $\left(\mathrm{H}_{2}\right)$ and the following conditions hold: 
$\left(H_{3}\right) \quad|f(t, x)| \leq p(t) \psi(|x|)$ for each $(t, x) \in[a, b] \times \mathbb{R}$, where $\psi:[0, \infty) \longrightarrow(0, \infty)$ is a continuous nondecreasing function and $p \in C\left([a, b], \mathbb{R}^{+}\right)$

$\left(H_{4}\right)$ There exists a constant $M>0$, such that

$$
\frac{\left(1-\Omega_{2}\right) M}{\psi(M)\|p\| \Omega_{1}}>1
$$

where $\Omega_{1}, \Omega_{2}$ are respectively given by (22) and (23).

Then, there exists at least one solution for problem (1)-(2) on $[a, b]$.

Proof. Let us verify that operator $\mathscr{A}$ defined by (21) satisfies the hypothesis of the Leray-Schauder Nonlinear Alternative [25]. In our first step, we establish that operator $\mathscr{A}$ maps bounded sets (balls) into a bounded set in $\mathscr{C}$. For a number $r>0$, let $B_{r}=\{x \in \mathscr{C}:\|x\| \leq r\}$ be a bounded ball in $\mathscr{C}$. Then, for $t \in[a, b]$, we have

$$
\begin{aligned}
& |(\mathscr{A} x)(t)| \leq \sup _{t \in[a, b]}\left\{I^{\alpha_{1}+\alpha_{2}}|f(t, x(t))|+|\lambda| I^{\alpha_{2}}|x(t)|\right. \\
& +\frac{(t-a)^{\gamma_{1}+\alpha_{2}-1}}{|\Lambda| \Gamma\left(\gamma_{1}+\alpha_{2}\right)}\left[|\theta| I^{\alpha_{1}+\alpha_{2}}|f(\eta, x(\eta))|+|\lambda||\theta| I^{\alpha_{2}}|x(\eta)|\right. \\
& \left.\left.+I^{\alpha_{1}+\alpha_{2}}|f(b, x(b))|+|\lambda| I^{\alpha_{2}}|x(b)|\right]\right\} \leq\|p\| \psi(\|x\|) \\
& \quad \cdot\left\{\frac{(b-a)^{\alpha_{1}+\alpha_{2}}}{\Gamma\left(\alpha_{1}+\alpha_{2}+1\right)}+\frac{(b-a)^{\gamma_{1}+\alpha_{2}-1}}{|\Lambda| \Gamma\left(\gamma_{1}+\alpha_{2}\right)}\left[\frac{(b-a)^{\alpha_{1}+\alpha_{2}}}{\Gamma\left(\alpha_{1}+\alpha_{2}+1\right)}\right.\right. \\
& \left.\left.+|\theta| \frac{(\eta-a)^{\alpha_{1}+\alpha_{2}}}{\Gamma\left(\alpha_{1}+\alpha_{2}+1\right)}\right]\right\} \\
& +\|x\|\left\{|\lambda| \frac{(b-a)^{\alpha_{2}}}{\Gamma\left(\alpha_{2}+1\right)}+\frac{(b-a)^{\gamma_{1}+\alpha_{2}-1}}{|\Lambda| \Gamma\left(\gamma_{1}+\alpha_{2}\right)}\right. \\
& \left.\quad \cdot\left[|\lambda| \frac{(b-a)^{\alpha_{2}}}{\Gamma\left(\alpha_{2}+1\right)}+|\lambda||\theta| \frac{(\eta-a)^{\alpha_{2}}}{\Gamma\left(\alpha_{2}+1\right)}\right]\right\} \\
& \quad=\|p\| \psi(\|x\|) \Omega_{1}+\|x\| \Omega_{2},
\end{aligned}
$$

and consequently,

$$
\|\mathscr{A} x\| \leq\|p\| \psi(r) \Omega_{1}+r \Omega_{2} .
$$

Next, we will show that $\mathscr{A}$ maps bounded sets into equicontinuous sets of $\mathscr{C}$. Let $\tau_{1}, \tau_{2} \in[a, b]$ with $\tau_{1}<\tau_{2}$ and $x \in B_{r}$. Then we have

$$
\begin{aligned}
& \left|(\mathscr{A} x)\left(\tau_{2}\right)-(\mathscr{A} x)\left(\tau_{1}\right)\right| \leq \frac{\|p\| \psi(r)}{\Gamma\left(\alpha_{1}+\alpha_{2}+1\right)}\left[\left(\tau_{2}-a\right)^{\alpha_{1}+\alpha_{2}}\right. \\
& \left.-\left(\tau_{1}-a\right)^{\alpha_{1}+\alpha_{2}}\right]+\frac{|\lambda| r}{\Gamma\left(\alpha_{2}+1\right)}\left(\tau_{2}-\tau_{1}\right)^{\alpha_{2}} \\
& +\frac{\left[\left(\tau_{2}-a\right)^{\gamma_{1}+\alpha_{2}-1}-\left(\tau_{1}-a\right)^{\gamma_{1}+\alpha_{2}-1}\right]}{|\Lambda| \Gamma\left(\gamma+\alpha_{2}\right)} \\
& \cdot\left[|\theta| \frac{(\eta-a)^{\alpha_{1}+\alpha_{2}}}{\Gamma\left(\alpha_{1}+\alpha_{2}+1\right)}\|p\| \psi(r)+|\lambda||\theta| \frac{(\eta-a)^{\alpha_{2}}}{\Gamma\left(\alpha_{2}+1\right)}\|p\| \psi(r)\right. \\
& \left.+\frac{(b-a)^{\alpha_{1}+\alpha_{2}}}{\Gamma\left(\alpha_{1}+\alpha_{2}+1\right)}\|p\| \psi(r)+|\lambda| \frac{(b-a)^{\alpha_{2}}}{\Gamma\left(\alpha_{2}+1\right)}\right] .
\end{aligned}
$$

Observe that the right-hand side of the above inequality tends to zero independently of $x \in B_{r}$ as $\tau_{2}-\tau_{1} \longrightarrow 0$. Thus, the set $\mathscr{A} B_{r}$ is equicontinuous. Therefore, the Arzelá-Ascoli theorem applies and hence operator $\mathscr{A}$ is completely continuous.

Finally, we show that the set of all solutions to equations $x=\lambda \mathscr{A} x$ is bounded for $\lambda \in(0,1)$.

Following the computation in the first step, we obtain

$$
|x(t)| \leq \psi(\|x\|)\|p\| \Omega_{1}+\|x\| \Omega_{2}, \quad t \in[a, b],
$$

which yields

$$
\frac{\left(1-\Omega_{2}\right)\|x\|}{\psi(\|x\|)\|p\| \Omega_{1}} \leq 1
$$

According to $\left(\mathrm{H}_{4}\right)$, there exists $M>0$ satisfying $\|x\| \neq M$. Introduce a set

$$
U=\{x \in C([a, b], \mathbb{R}):\|x\|<M\},
$$

and notice that $\mathscr{A}: \bar{U} \longrightarrow \mathscr{C}$ is continuous and completely continuous. Then, the choice of $U$ implies that there is no $x \in \partial U$, such that $x=\lambda \mathscr{A} x$ for some $\lambda \in(0,1)$. In consequence, we deduce by the nonlinear alternative of the Leray-Schauder type [25] that $\mathscr{A}$ has a fixed-point $x \in \bar{U}$, which corresponds to a solution of problem (1)-(2). This completes the proof.

Example 11. Consider the three-point boundary value problem of the Langevin equation with the Hilfer fractional derivative of the form:

$$
\left\{\begin{array}{l}
{ }^{H} D^{(4 / 7),(1 / 3)}\left({ }^{H} D^{(2 / 5),(4 / 5)}+\frac{1}{5}\right) x(t)=\frac{1}{80}(4 t+1)\left(\frac{x^{8}(t)}{x^{6}(t)+1}+\frac{1}{4}\right), \quad t \in\left[\frac{1}{4}, \frac{3}{4}\right], \\
x\left(\frac{1}{4}\right)=0, \quad x\left(\frac{3}{4}\right)=\frac{2}{3} x\left(\frac{1}{2}\right) .
\end{array}\right.
$$

Here $\alpha_{1}=4 / 7, \alpha_{2}=2 / 5, \beta_{1}=1 / 3, \beta_{2}=4 / 5, \lambda=1 / 5, a=1 / 4$, $b=3 / 4, \eta=1 / 2$, and $\theta=2 / 3$. Using the given values, we obtain $\gamma_{1}=5 / 7, \quad \gamma_{2}=22 / 25, \Lambda \approx 0.3752096355, \Omega_{1} \approx 2.316402992$, and $\Omega_{2} \approx 0.8402809682$. Next, the nonlinear function is bounded as

$$
\begin{aligned}
f(t, x) & =\frac{1}{80}(4 t+1)\left(\frac{x^{8}}{x^{6}+1}+\frac{1}{4}\right) \leq \frac{1}{80}(4 t+1)\left(x^{2}+\frac{1}{4}\right) \\
& :=p(t) \psi(x)
\end{aligned}
$$

which satisfies condition $\mathrm{H}_{4}$ with $\|p\|=1 / 20$ and $\psi(\mathrm{M})=M^{2}$ $+(1 / 4)$. Furthermore, we find that $M \in(0.2147201605$, 1.164306134), satisfying $\mathrm{H}_{4}$ of Theorem 10 . Therefore, by applying Theorem 10, the boundary value problem (38) has at least one solution on $[1 / 4,3 / 4]$. 
In the following result, we apply Banach's fixed-point theorem to prove the existence of a unique solution of the problem at hand.

\section{Theorem 12. Assume that}

$\left(H_{5}\right)|f(t, x)-f(t, y)| \leq L|x-y|, L>0$ for each $t \in[a, b]$ and $x, y \in \mathbb{R}$.

If the constants $\Omega_{1}, \Omega_{2}$ defined by (22) and (23), respectively, are such that

$$
L \Omega_{1}+\Omega_{2}<1,
$$

then problem (1)-(2) has a unique solution on $[a, b]$.

Proof. Let us first show that $\mathscr{A}$ defined by (21) satisfies $\mathscr{A} B_{r} \subset B_{r}$, where $B_{r}=\{x \in \mathscr{C}:\|x\| \leq r\}$ with $r \geq\left(M \Omega_{1} /(1\right.$ $\left.\left.-L \Omega_{1}-\Omega_{2}\right)\right)$ and $\sup _{t \in[a, b]}|f(t, 0)|=M<\infty$. For any $x \in$ $B_{r}$, we have

$$
\begin{aligned}
& |(\mathscr{A} x)(t)| \leq \sup _{t \in[a, b]}\left\{I^{\alpha_{1}+\alpha_{2}}|f(t, x(t))|+|\lambda| I^{\alpha_{2}}|x(t)|\right. \\
& +\frac{(t-a)^{\gamma_{1}+\alpha_{2}-1}}{|\Lambda| \Gamma\left(\gamma_{1}+\alpha_{2}\right)}\left[|\theta| I^{\alpha_{1}+\alpha_{2}}|f(\eta, x(\eta))|+|\lambda||\theta| I^{\alpha_{2}}|x(\eta)|\right. \\
& \left.\left.+I^{\alpha_{1}+\alpha_{2}}|f(b, x(b))|+|\lambda| I^{\alpha_{2}}|x(b)|\right]\right\} \leq(L|| x||+M) \\
& \quad+\left\{\frac{(b-a)^{\alpha_{1}+\alpha_{2}}}{\Gamma\left(\alpha_{1}+\alpha_{2}+1\right)}+\frac{(b-a)^{\gamma_{1}+\alpha_{2}-1}}{|\Lambda| \Gamma\left(\gamma_{1}+\alpha_{2}\right)}\left[\frac{(b-a)^{\alpha_{1}+\alpha_{2}}}{\Gamma\left(\alpha_{1}+\alpha_{2}+1\right)}\right.\right. \\
& \left.\left.+|\theta| \frac{(\eta-a)^{\alpha_{1}+\alpha_{2}}}{\Gamma\left(\alpha_{1}+\alpha_{2}+1\right)}\right]\right\}+\| x||\left\{|\lambda| \frac{(b-a)^{\alpha_{2}}}{\Gamma\left(\alpha_{2}+1\right)}\right. \\
& \left.+\frac{(b-a)^{\gamma_{1}+\alpha_{2}-1}}{|\Lambda| \Gamma\left(\gamma_{1}+\alpha_{2}\right)}\left[|\lambda| \frac{(b-a)^{\alpha_{2}}}{\Gamma\left(\alpha_{2}+1\right)}+|\lambda||\theta| \frac{(\eta-a)^{\alpha_{2}}}{\Gamma\left(\alpha_{2}+1\right)}\right]\right\} \\
& \quad \leq(L r+M) \Omega_{1}+r \Omega_{2}<r,
\end{aligned}
$$

which implies that $\mathscr{A} B_{r} \subset B_{r}$.

Next, we let $x, y \in \mathscr{C}$. Then for $t \in[a, b]$, we have

$$
\begin{aligned}
& |(\mathscr{A} x)(t)-(\mathscr{A} y)(t)| \\
& \quad \leq\left\{\frac{(b-a)^{\alpha_{1}+\alpha_{2}}}{\Gamma\left(\alpha_{1}+\alpha_{2}+1\right)}+\frac{(b-a)^{\gamma_{1}+\alpha_{2}-1}}{|\Lambda| \Gamma\left(\gamma_{1}+\alpha_{2}\right)}\left[\frac{(b-a)^{\alpha_{1}+\alpha_{2}}}{\Gamma\left(\alpha_{1}+\alpha_{2}+1\right)}\right.\right. \\
& \left.\left.\quad+|\theta| \frac{(\eta-a)^{\alpha_{1}+\alpha_{2}}}{\Gamma\left(\alpha_{1}+\alpha_{2}+1\right)}\right]\right\}\|x-y\| \\
& \quad+\left\{|\lambda| \frac{(b-a)^{\alpha_{2}}}{\Gamma\left(\alpha_{2}+1\right)}+\frac{(b-a)^{\gamma_{1}+\alpha_{2}-1}}{|\Lambda| \Gamma\left(\gamma_{1}+\alpha_{2}\right)}\left[|\lambda| \frac{(b-a)^{\alpha_{2}}}{\Gamma\left(\alpha_{2}+1\right)}\right.\right. \\
& \left.\left.\quad+|\lambda||\theta| \frac{(\eta-a)^{\alpha_{2}}}{\Gamma\left(\alpha_{2}+1\right)}\right]\right\}\|x-y\|=\left(L \Omega_{1}+\Omega_{2}\right)\|x-y\|,
\end{aligned}
$$

which implies that $\|\mathscr{A} x-\mathscr{A} y\| \leq\left(L \Omega_{1}+\Omega_{2}\right)\|x-y\|$. As $L$ $\Omega_{1}+\Omega_{2}<1, \mathscr{A}$ is a contraction. Therefore, by Banach's fixed-point theorem, operator $\mathscr{A}$ has a fixed point which is indeed a unique solution of problem (1)-(2). The proof is finished.

Example 13. Consider the three-point boundary value problem of the Langevin equation with the Hilfer fractional derivative of the form:

$$
\left\{\begin{array}{l}
{ }^{H} D^{(1 / 2),(3 / 5)}\left({ }^{H} D^{(3 / 4),(2 / 5)}+\frac{2}{17}\right) x(t)=\frac{2 e^{(1 / 2)-t}}{159+2 t}\left(\frac{x^{2}(t)+2|x(t)|}{1+|x(t)|}\right)+\frac{2}{3}, \quad t \in\left[\frac{1}{2}, \frac{5}{2}\right], \\
x\left(\frac{1}{2}\right)=0, \quad x\left(\frac{5}{2}\right)=\frac{5}{8} x\left(\frac{3}{2}\right) .
\end{array}\right.
$$

Here, $\alpha_{1}=1 / 2, \alpha_{2}=3 / 4, \beta_{1}=3 / 5, \beta_{2}=2 / 5, \lambda=2 / 17, a=$ $1 / 2, b=5 / 2, \eta=3 / 2, \theta=5 / 8$, and

$$
f(t, x)=\frac{2 e^{(1 / 2)-t}}{159+2 t}\left(\frac{x^{2}+2|x|}{1+|x|}\right)+\frac{2}{3} .
$$

Using the given data, we obtain $\gamma_{1}=4 / 5, \gamma_{2}=17 / 20, \Lambda$ $\approx 0.9439932223, \quad \Omega_{1} \approx 6.724559445, \quad \Omega_{2} \approx 0.7305167191$, and $L=1 / 40$ as

$$
|f(t, x)-f(t, y)| \leq \frac{4 e^{(1 / 2)-t}}{159+2 t}|x-y| \leq \frac{1}{40}|x-y| .
$$

Moreover, we have $L \Omega_{1}+\Omega_{2} \approx 0.8986307052<1$. As all the assumptions of Theorem 12 are satisfied, we therefore deduce by its conclusion that problem (43) has a unique solution on $[1 / 2,5 / 2]$.

\section{Existence Results for Multivalued Problems (3) and (4)}

Definition 14. A continuous function $x$ is said to be a solution of problem (3)-(4) if $x(a)=0, x(b)=\theta x(\eta)$ and there exists a function $v \in L^{1}([a, b], \mathbb{R})$ with $v \in F(t, x)$, a.e., on $[a, b]$ such that

$$
\begin{aligned}
x(t)= & I^{\alpha_{1}+\alpha_{2}} v(t)-\lambda I^{\alpha_{2}} x(t) \\
& +\frac{(t-a)^{\gamma_{1}+\alpha_{2}-1}}{\Lambda \Gamma\left(\gamma_{1}+\alpha_{2}\right)}\left[\theta I^{\alpha_{1}+\alpha_{2}} v(\eta)-\lambda \theta I^{\alpha_{2}} x(\eta)-I^{\alpha_{1}+\alpha_{2}} v(b)\right. \\
& \left.+\lambda I^{\alpha_{2}} x(b)\right] .
\end{aligned}
$$

For each $y \in C([a, b], \mathbb{R})$, define the set of selections of $F$ by

$$
S_{F, x}:=\left\{v \in L^{1}([a, b], \mathbb{R}): v \in F(t, x(t)) \text { on }[a, b]\right\} .
$$

Lemma 15 (see [27]). Let $X$ be a separable Banach space. Let $F:[a, b] \times \mathbb{R} \longrightarrow \mathscr{P}_{c p, c}(X)$ be an $L^{1}$-multivalued map and let $\Theta$ be a linear continuous mapping from $L^{1}([a, b], X)$ to $C([a, b], X)$. Then the operator 


$$
\begin{array}{r}
\Theta \circ S_{F}: C([a, b], X) \rightarrow \mathscr{P}_{c p, c}(C([a, b], X)), \\
x \mapsto\left(\Theta \circ S_{F}\right)(x)=\Theta\left(S_{F, x}\right),
\end{array}
$$

is a closed graph operator in $C([a, b], X) \times C([a, b], X)$.

Our first existence result, dealing with the convex valued $F$, is based on the nonlinear alternative of the Leray-Schauder type for (Kakutani) multivalued maps [25] with the assumption that $F$ is Carathéodory.

Theorem 16. Suppose that $\left(\mathrm{H}_{2}\right),\left(\mathrm{H}_{4}\right)$, and the following conditions hold:

$\left(A_{1}\right) \quad F:[a, b] \times \mathbb{R} \longrightarrow \mathscr{P}_{c p, c}(\mathbb{R})$ is $L^{1}$-Carathéodory, where $\mathscr{P}_{c p, c}(\mathbb{R})=\{Y \in \mathscr{P}(\mathbb{R}): Y$ is compact and convex $\}$

$\left(A_{2}\right)\|F(t, x)\|_{\mathscr{P}}:=\sup \{|y|: y \in F(t, x)\} \leq p(t) \psi(|x|)$ for each $(t, x) \in[a, b] \times \mathbb{R}$, where $\psi:[0, \infty) \longrightarrow(0, \infty)$ is a continuous nondecreasing function and $p \in C\left([a, b], \mathbb{R}^{+}\right)$

Then, there exists at least one solution for problem (3)-(4) on $[a, b]$.

Proof. Let us transform problem (3)-(4) into a fixed-point problem by introducing an operator $\mathscr{F}: C([a, b], \mathbb{R}) \longrightarrow \mathscr{P}$ $(C([a, b], \mathbb{R}))$ as

$$
\begin{aligned}
& \mathscr{F}(x) \\
& \quad=\left\{h \in C([a, b], \mathbb{R}): h(t)=\left\{I^{\alpha_{1}+\alpha_{2}} v(t)-\lambda I^{\alpha_{2}} x(t)\right.\right. \\
& \quad+\frac{(t-a)^{\gamma_{1}+\alpha_{2}-1}}{\Lambda \Gamma\left(\gamma_{1}+\alpha_{2}\right)}\left[\theta I^{\alpha_{1}+\alpha_{2}} v(\eta)-\lambda \theta I^{\alpha_{2}} x(\eta)-I^{\alpha_{1}+\alpha_{2}} v(b)\right. \\
& \left.\left.\quad+\lambda I^{\alpha_{2}} x(b)\right]\right\},
\end{aligned}
$$

for $t \in[a, b]$ and $v \in S_{F, x}$. Notice that the existence of a fixed point of $\mathscr{F}$ ensures the existence of a solution of problems (1) and (2). This will be achieved by establishing that operator $\mathscr{F}$ satisfies the hypothesis of the Leray-Schauder nonlinear alternative for the Kakutani maps [25]. We do it in several steps.

Step 1. Since $S_{F, x}$ is convex ( $F$ has convex values), therefore $\mathscr{F}(x)$ is convex for each $x \in C([a, b], \mathbb{R})$.

Step 2. $\mathscr{F}$ maps bounded sets (balls) into bounded sets in $C$ $([a, b], \mathbb{R})$.

Let $B_{r}=\{x \in C([a, b], \mathbb{R}):\|x\| \leq r\}$ be a bounded set in $C([a, b], \mathbb{R})$. Then, for each $h \in \mathscr{B}(x), x \in B_{r}$, there exists $v$ $\in S_{F, x}$ such that

$$
\begin{aligned}
h(t)= & I^{\alpha_{1}+\alpha_{2}} v(t)-\lambda I^{\alpha_{2}} x(t) \\
& +\frac{(t-a)^{\gamma_{1}+\alpha_{2}-1}}{\Lambda \Gamma\left(\gamma_{1}+\alpha_{2}\right)}\left[\theta I^{\alpha_{1}+\alpha_{2}} v(\eta)-\lambda \theta I^{\alpha_{2}} x(\eta)-I^{\alpha_{1}+\alpha_{2}} v(b)\right. \\
& \left.+\lambda I^{\alpha_{2}} x(b)\right] .
\end{aligned}
$$

Then, for $t \in[a, b]$, we have

$$
\begin{aligned}
& |h(t)| \leq \sup _{t \in[a, b]}\left\{I^{\alpha_{1}+\alpha_{2}}|v(t)|+|\lambda| I^{\alpha_{2}}|x(t)|\right. \\
& \quad+\frac{(t-a)^{\gamma_{1}+\alpha_{2}-1}}{|\Lambda| \Gamma\left(\gamma_{1}+\alpha_{2}\right)}\left[\left.|\theta|\right|^{\alpha_{1}+\alpha_{2}}|v(\eta)|+|\lambda||\theta| I^{\alpha_{2}}|x(\eta)|\right. \\
& \left.\left.\quad+I^{\alpha_{1}+\alpha_{2}}|v(b)|+|\lambda| I^{\alpha_{2}}|x(b)|\right]\right\} \leq\|p\| \psi \psi(\|x\|) \\
& \quad+\left\{\frac{(b-a)^{\alpha_{1}+\alpha_{2}}}{\Gamma\left(\alpha_{1}+\alpha_{2}+1\right)}+\frac{(b-a)^{\gamma_{1}+\alpha_{2}-1}}{|\Lambda| \Gamma\left(\gamma_{1}+\alpha_{2}\right)}\left[\frac{(b-a)^{\alpha_{1}+\alpha_{2}}}{\Gamma\left(\alpha_{1}+\alpha_{2}+1\right)}\right.\right. \\
& \left.\left.\quad+|\theta| \frac{(\eta-a)^{\alpha_{1}+\alpha_{2}}}{\Gamma\left(\alpha_{1}+\alpha_{2}+1\right)}\right]\right\} \\
& \quad+\|x\|\left\{|\lambda| \frac{(b-a)^{\alpha_{2}}}{\Gamma\left(\alpha_{2}+1\right)}+\frac{(b-a)^{\gamma_{1}+\alpha_{2}-1}}{|\Lambda| \Gamma\left(\gamma_{1}+\alpha_{2}\right)}\left[|\lambda| \frac{(b-a)^{\alpha_{2}}}{\Gamma\left(\alpha_{2}+1\right)}\right.\right. \\
& \left.\left.\quad+|\lambda||\theta| \frac{(\eta-a)^{\alpha_{2}}}{\Gamma\left(\alpha_{2}+1\right)}\right]\right\}=\|p\| \psi(\|x\|) \Omega_{1}+\|x\| \Omega_{2} .
\end{aligned}
$$

Thus,

$$
\|h\| \leq\|p\| \psi(r) \Omega_{1}+r \Omega_{2} .
$$

Step 3. $\mathscr{T}$ maps bounded sets into equicontinuous sets of $C$ $([a, b], \mathbb{R})$.

Let $\tau_{1}, \tau_{2} \in[a, b]$ with $\tau_{1}<\tau_{2}$ and $x \in B_{r}$. Then, for each $h \in \mathscr{B}(x)$, we obtain

$$
\begin{aligned}
& \left|h\left(t_{2}\right)-h\left(t_{1}\right)\right| \leq \frac{1}{\Gamma\left(\alpha_{1}+\alpha_{2}\right)} \\
& \quad \cdot \mid \int_{a}^{\tau_{1}}\left[\left(\tau_{2}-s\right)^{\alpha_{1}+\alpha_{2}-1}-\left(\tau_{1}-s\right)^{\alpha_{1}+\alpha_{2}-1}\right] v(s) d s \\
& \quad+\int_{\tau_{1}}^{t_{2}}\left(\tau_{2}-s\right)^{\alpha_{1}+\alpha_{2}-1} v(s) d s \mid \\
& \quad+\mid \frac{1}{\Gamma\left(\alpha_{2}\right)} \int_{a}^{\tau_{1}}\left[\left(\tau_{2}-s\right)^{\alpha_{2}-1}-\left(\tau_{1}-s\right)^{\alpha_{2}-1}\right] \\
& \quad \cdot x(s) d s+\frac{1}{\Gamma\left(\alpha_{2}\right)} \int_{\tau_{1}}^{\tau_{2}}\left(\tau_{2}-s\right)^{\alpha_{2}-1} x(s) d s \mid \\
& \quad+\frac{\left(\tau_{2}-a\right)^{\gamma_{1}+\alpha_{2}-1}-\left(\tau_{1}-a\right)^{\gamma_{1}+\alpha_{2}-1}}{\Gamma\left(\gamma+\alpha_{2}\right)}\left[|\lambda||\theta| I^{\alpha_{2}}|x(\eta)|\right. \\
& \left.\quad+|\lambda| I^{\alpha_{2}}|x(b)|\right]+\frac{\left(\tau_{2}-a\right)^{\gamma_{1}+\alpha_{2}-1}-\left(\tau_{1}-a\right)^{\gamma_{1}+\alpha_{2}-1}}{|\Lambda| \Gamma\left(\gamma_{1}+\alpha_{2}\right)} \\
& \quad \cdot\left[|\theta| \frac{(\eta-a)^{\alpha_{1}+\alpha_{2}}}{\Gamma\left(\alpha_{1}+\alpha_{2}+1\right)}+\frac{(b-a)^{\alpha_{1}+\alpha_{2}}}{\Gamma\left(\alpha_{1}+\alpha_{2}+1\right)}\right]\|p\| \psi \psi(r) \\
& \leq \frac{\|p\| \psi(r)}{\Gamma\left(\alpha_{1}+\alpha_{2}\right)}\left[2\left(\tau_{2}-\tau_{1}\right)^{\alpha_{1}+\alpha_{2}}+\left|\left(\tau_{2}-a\right)^{\alpha_{1}+\alpha_{2}}-\left(\tau_{1}-a\right)^{\alpha_{1}+\alpha_{2}}\right|\right] \\
& \quad+\frac{r}{\Gamma\left(\alpha_{2}\right)}\left[2\left(\tau_{2}-\tau_{1}\right)^{\alpha_{2}}+\left|\left(\tau_{2}-a\right)^{\alpha_{2}}-\left(\tau_{1}-a\right)^{\alpha_{2}}\right|\right] \\
& \quad+\frac{\left(\tau_{2}-a\right)^{\gamma_{1}+\alpha_{2}-1}-\left(\tau_{1}-a\right)^{\gamma_{1}+\alpha_{2}-1}}{\Gamma\left(\gamma+\alpha_{2}\right)}\left[|\lambda||\theta| I^{\alpha_{2}}|x(\eta)|\right. \\
& \quad+|\lambda| I^{\alpha_{2}}|x(b)| \mid r+\frac{\left(\tau_{2}-a\right)^{\gamma_{1}+\alpha_{2}-1}-\left(\tau_{1}-a\right)^{\gamma_{1}+\alpha_{2}-1}}{|\Lambda| \Gamma\left(\gamma_{1}+\alpha_{2}\right)} \\
& \quad \cdot\left[|\theta| \frac{(\eta-a)^{\alpha_{1}+\alpha_{2}}}{\Gamma\left(\alpha_{1}+\alpha_{2}+1\right)}+\frac{(b-a)^{\alpha_{1}+\alpha_{2}}}{\Gamma\left(\alpha_{1}+\alpha_{2}+1\right)}\right] \\
& \cdot\|p\| \psi(r) \longrightarrow 0 \text { as } \tau_{2}-\tau_{1} \longrightarrow 0 \text { independently of } x \in B_{r} .
\end{aligned}
$$


Therefore, $\quad \mathscr{F}: C([a, b], \mathbb{R}) \longrightarrow \mathscr{P}(C([a, b], \mathbb{R})) \quad$ is completely continuous by the application of the ArzeláAscoli theorem.

Next, we show that $\mathscr{F}$ is upper semicontinuous by proving that it has a closed graph ([28], Proposition 1.2) as $\mathscr{F}$ is already shown to be completely continuous.

Step 4. $\mathscr{F}$ has a closed graph.

Let $x_{n} \longrightarrow x_{*}, h_{n} \in \mathscr{F}\left(x_{n}\right)$ and $h_{n} \longrightarrow h_{*}$. Then, we need to show that $h_{*} \in \mathscr{F}\left(x_{*}\right)$. Associated with $h_{n} \in \mathscr{F}\left(x_{n}\right)$, there exists $v_{n} \in S_{F, x_{n}}$ such that for each $t \in[a, b]$,

$$
\begin{aligned}
h(t)= & I^{\alpha_{1}+\alpha_{2}} v_{n}(t)-\lambda I^{\alpha_{2}} x(t) \\
& +\frac{(t-a)^{\gamma_{1}+\alpha_{2}-1}}{\Lambda \Gamma\left(\gamma_{1}+\alpha_{2}\right)}\left[\theta I^{\alpha_{1}+\alpha_{2}} v_{n}(\eta)-\lambda \theta I^{\alpha_{2}} x(\eta)\right. \\
& \left.-I^{\alpha_{1}+\alpha_{2}} v_{n}(b)+\lambda I^{\alpha_{2}} x(b)\right] .
\end{aligned}
$$

Thus, it suffices to show that there exists $v_{*} \in S_{F, x_{*}}$, such that for each $t \in[a, b]$,

$$
\begin{aligned}
h_{*}(t)= & I^{\alpha_{1}+\alpha_{2}} v_{*}(t)-\lambda I^{\alpha_{2}} x(t) \\
& +\frac{(t-a)^{\gamma_{1}+\alpha_{2}-1}}{\Lambda \Gamma\left(\gamma_{1}+\alpha_{2}\right)}\left[\theta I^{\alpha_{1}+\alpha_{2}} v_{*}(\eta)-\lambda \theta I^{\alpha_{2}} x(\eta)\right. \\
& \left.-I^{\alpha_{1}+\alpha_{2}} v_{*}(b)+\lambda I^{\alpha_{2}} x(b)\right] .
\end{aligned}
$$

Let us introduce the linear operator $\Theta: L^{1}([a, b], \mathbb{R})$ $\longrightarrow C([a, b], \mathbb{R})$ as by

$$
\begin{aligned}
v \mapsto \Theta(v)(t)= & I^{\alpha_{1}+\alpha_{2}} v(t)-\lambda I^{\alpha_{2}} x(t) \\
& +\frac{(t-a)^{\gamma_{1}+\alpha_{2}-1}}{\Lambda \Gamma\left(\gamma_{1}+\alpha_{2}\right)}\left[\theta I^{\alpha_{1}+\alpha_{2}} v(\eta)-\lambda \theta I^{\alpha_{2}} x(\eta)\right. \\
& \left.-I^{\alpha_{1}+\alpha_{2}} v(b)+\lambda I^{\alpha_{2}} x(b)\right] .
\end{aligned}
$$

Observe that $\left\|h_{n}-h_{*}\right\| \rightarrow 0$, as $n \rightarrow \infty$. So, by Lemma 15, $\Theta \circ S_{F}$ is a closed graph operator. Furthermore, we have $h_{n}(t) \in \Theta\left(S_{F, x_{n}}\right)$. Since $x_{n} \rightarrow x_{*}$, we have

$$
\begin{aligned}
h_{*}(t)= & I^{\alpha_{1}+\alpha_{2}} v_{*}(t)-\lambda I^{\alpha_{2}} x(t) \\
& +\frac{(t-a)^{\gamma_{1}+\alpha_{2}-1}}{\Lambda \Gamma\left(\gamma_{1}+\alpha_{2}\right)}\left[\theta I^{\alpha_{1}+\alpha_{2}} v_{*}(\eta)-\lambda \theta I^{\alpha_{2}} x(\eta)\right. \\
& \left.-I^{\alpha_{1}+\alpha_{2}} v_{*}(b)+\lambda I^{\alpha_{2}} x(b)\right]
\end{aligned}
$$

for some $v_{*} \in S_{F, x_{*}}$.

Step 5. We show that there exists an open set $U \subseteq C([a, b], \mathbb{R})$ with $x \notin \nu \mathscr{F}(x)$ for any $v \in(0,1)$ and all $x \in \partial U$.
Let $v \in(0,1)$ and $x \in v \mathscr{F}(x)$. Then, there exists $v \in L^{1}([a$, $b], \mathbb{R})$ with $v \in S_{F, x}$, such that for $t \in[a, b]$, we have

$$
\begin{aligned}
x(t)= & I^{\alpha_{1}+\alpha_{2}} v(t)-\lambda I^{\alpha_{2}} x(t) \\
& +\frac{(t-a)^{\gamma_{1}+\alpha_{2}-1}}{\Lambda \Gamma\left(\gamma_{1}+\alpha_{2}\right)}\left[\theta I^{\alpha_{1}+\alpha_{2}} v(\eta)-\lambda \theta I^{\alpha_{2}} x(\eta)\right. \\
& \left.-I^{\alpha_{1}+\alpha_{2}} v(b)+\lambda I^{\alpha_{2}} x(b)\right] .
\end{aligned}
$$

As in the second step, it can be shown that

$$
\begin{aligned}
& |x(t)| \leq\|p\| \psi(\|x\|) \\
& \cdot\left\{\frac{(b-a)^{\alpha_{1}+\alpha_{2}}}{\Gamma\left(\alpha_{1}+\alpha_{2}+1\right)}+\frac{(b-a)^{\gamma_{1}+\alpha_{2}-1}}{|\Lambda| \Gamma\left(\gamma_{1}+\alpha_{2}\right)}\right. \\
& \left.\cdot\left[\frac{(b-a)^{\alpha_{1}+\alpha_{2}}}{\Gamma\left(\alpha_{1}+\alpha_{2}+1\right)}+|\theta| \frac{(\eta-a)^{\alpha_{1}+\alpha_{2}}}{\Gamma\left(\alpha_{1}+\alpha_{2}+1\right)}\right]\right\} \\
& +\|x\|\left\{|\lambda| \frac{(b-a)^{\alpha_{2}}}{\Gamma\left(\alpha_{2}+1\right)}+\frac{(b-a)^{\gamma_{1}+\alpha_{2}-1}}{|\Lambda| \Gamma\left(\gamma_{1}+\alpha_{2}\right)}\right. \\
& \left.\cdot\left[|\lambda| \frac{(b-a)^{\alpha_{2}}}{\Gamma\left(\alpha_{2}+1\right)}+|\lambda \| \theta| \frac{(\eta-a)^{\alpha_{2}}}{\Gamma\left(\alpha_{2}+1\right)}\right]\right\} \\
& =\|p\| \psi(\|x\|) \Omega_{1}+\|x\| \Omega_{2},
\end{aligned}
$$

which implies that

$$
\|x\| \leq\|p\| \psi(\|x\|) \Omega_{1}+\|x\| \Omega_{2} .
$$

\section{Consequently}

$$
\frac{\left(1-\Omega_{2}\right)\|x\|}{\|p\| \psi(\|x\|) \Omega_{1}} \leq 1
$$

By $\mathrm{H}_{4}$, we can find a number $M$ with $\|x\| \neq M$. Define

$$
U=\{x \in C([a, b], \mathbb{R}):\|x\|<M\} .
$$

Notice that operator $\mathscr{F}: \bar{U} \longrightarrow \mathscr{P}(C([a, b], \mathbb{R}))$ is compact, upper semicontinuous, and convex valued. By the choice of $U$, we cannot find $x \in \partial U$ satisfying $x \in \nu \mathscr{F}(x)$ for some $v$ $\epsilon(0,1)$. Therefore, by the Leray-Schauder nonlinear alternative for the Kakutani maps [25], $\mathscr{F}$ has a fixed point $y \in \bar{U}$ which is a solution of problem (3)-(4). This completes the proof.

In our next result, we show the existence of solutions for the nonconvex valued case of problem (3)-(4). For that, we need the following assumptions.

$\left(\mathrm{B}_{1}\right) F:[a, b] \times \mathbb{R} \longrightarrow \mathscr{P}_{c p}(\mathbb{R})$ is such that $F(\cdot, x):[a, b]$ $\longrightarrow \mathscr{P}_{c p}(\mathbb{R})$ is measurable for each $x \in \mathbb{R}$, where $\mathscr{P}_{c p}$ $(\mathbb{R})=\{Y \in \mathscr{P}(\mathbb{R}): Y$ is compact $\}$ 
$\left(\mathrm{B}_{2}\right) H_{d}(F(t, x), F(t, \bar{x})) \leq m(t)|x-\bar{x}|$ for almost all $t \in$ $[a, b]$ and $x, \bar{x} \in \mathbb{R}$ with $m \in C\left([a, b], \mathbb{R}^{+}\right)$and $d(0, F(t$, $0)) \leq m(t)$ for almost all $t \in[a, b]$

Recall that $H_{d}: \mathscr{P}(X) \times \mathscr{P}(X) \rightarrow \mathbb{R} \cup\{\infty\}$ is defined by

$$
H_{d}(\mathscr{V}, \mathscr{W})=\max \left\{\sup _{v \in \mathscr{V}} d(v, \mathscr{W}), \sup _{w \in \mathscr{W}} d(\mathscr{V}, w)\right\}
$$

where $d(\mathscr{V}, w)=\inf _{v \in \mathscr{V}} d(v ; w), d(v, \mathscr{W})=\inf _{w \in \mathscr{W}} d(v ; w)$, and $(X, d)$ is a metric space induced from the normed space $(X ;\|\cdot\|)$.

We apply a fixed-point theorem for multivalued maps due to Covitz and Nadler [29]: if $\mathscr{N}: X \longrightarrow \mathscr{P}_{c l}(X)$ is a contraction, then Fix $\mathcal{N} \neq \varnothing$, where $\mathscr{P}_{c l}(X)=\{Y \in \mathscr{P}(X): Y$ is closed $\}$.

Theorem 17. Suppose that conditions $\left(B_{1}\right)$ and $\left(B_{2}\right)$ hold and that

$$
\delta:=\|m\| \Omega_{1}+\Omega_{2}<1 .
$$

Then, problem (3)-(4) has at least one solution on $[a, b]$.

Proof. In view of $\left(\mathrm{B}_{1}\right)$, the set $S_{F, x}$ is nonempty for each $x \in$ $C([a, b], \mathbb{R})$, and hence $F$ has a measurable selection (see Theorem III.6 in [30]). Now, we verify that operator $\mathscr{F}$ defined by (49) satisfies the hypothesis of Covitz and Nadler's fixed theorem [29]. In order to establish that $\mathscr{F}(x) \in \mathscr{P}_{c l}$ $(C([a, b], \mathbb{R}))$ for each $x \in C([a, b], \mathbb{R})$, let $\left\{u_{n}\right\}_{n \geq 0} \in \mathscr{F}(x)$ be such that $u_{n} \longrightarrow u(n \longrightarrow \infty)$ in $C([a, b], \mathbb{R})$. Then, $u \in C([\mathrm{a}$, $b], \mathbb{R})$ and there exists $v_{n} \in S_{F, x_{n}}$ such that, for each $t \in[a, b]$

$$
\begin{aligned}
u_{n}(t)= & I^{\alpha_{1}+\alpha_{2}} v_{n}(t)-\lambda I^{\alpha_{2}} x(t) \\
& +\frac{(t-a)^{\gamma_{1}+\alpha_{2}-1}}{\Lambda \Gamma\left(\gamma_{1}+\alpha_{2}\right)}\left[\theta I^{\alpha_{1}+\alpha_{2}} v_{n}(\eta)-\lambda \theta I^{\alpha_{2}} x(\eta)\right. \\
& \left.-I^{\alpha_{1}+\alpha_{2}} v_{n}(b)+\lambda I^{\alpha_{2}} x(b)\right] .
\end{aligned}
$$

Since $F$ is compact valued, we pass onto a subsequence (if necessary) to obtain that $v_{n}$ converges to $v$ in $L^{1}([a, b], \mathbb{R})$. Therefore, $v \in S_{F, x}$, and for each $t \in[a, b]$, we have

$$
\begin{aligned}
u_{n}(t) \longrightarrow u(t)= & I^{\alpha_{1}+\alpha_{2}} v(t)-\lambda I^{\alpha_{2}} x(t) \\
& +\frac{(t-a)^{\gamma_{1}+\alpha_{2}-1}}{\Lambda \Gamma\left(\gamma_{1}+\alpha_{2}\right)}\left[\theta I^{\alpha_{1}+\alpha_{2}} v(\eta)-\lambda \theta I^{\alpha_{2}} x(\eta)\right. \\
& \left.-I^{\alpha_{1}+\alpha_{2}} v(b)+\lambda I^{\alpha_{2}} x(b)\right] .
\end{aligned}
$$

which implies that $u \in \mathscr{F}(x)$.

Next, we show that we can find a $\delta<1$ (defined by (64)) satisfying

$$
H_{d}(\mathscr{F}(x), \mathscr{F}(\bar{x})) \leq \delta\|x-\bar{x}\| \text { for each } x, \quad \bar{x} \in C^{2}([a, b], \mathbb{R}) .
$$

Let $x, \bar{x} \in C^{2}([a, b], \mathbb{R})$ and $h_{1} \in \mathscr{F}(x)$. Then, there exists $v_{1}(t) \in F(t, x(t))$ such that, for each $t \in[a, b]$,

$$
\begin{aligned}
h_{1}(t)= & I^{\alpha_{1}+\alpha_{2}} v_{1}(t)-\lambda I^{\alpha_{2}} x(t) \\
& +\frac{(t-a)^{\gamma_{1}+\alpha_{2}-1}}{\Lambda \Gamma\left(\gamma_{1}+\alpha_{2}\right)}\left[\theta I^{\alpha_{1}+\alpha_{2}} v_{1}(\eta)-\lambda \theta I^{\alpha_{2}} x(\eta)\right. \\
& \left.-I^{\alpha_{1}+\alpha_{2}} v_{1}(b)+\lambda I^{\alpha_{2}} x(b)\right] .
\end{aligned}
$$

By assumption $\left(\mathrm{B}_{2}\right)$, we have that $H_{d}(F(t, x), F(t, \bar{x})) \leq$ $m(t)|x(t)-\bar{x}(t)|$. So we can find $\mathrm{Q} \in F(t, \bar{x}(t))$, such that

$$
\left|v_{1}(t)-\varrho\right| \leq m(t)|x(t)-\bar{x}(t)|, \quad t \in[a, b] .
$$

Define $\mathcal{Y}:[a, b] \longrightarrow \mathscr{P}(\mathbb{R})$ by

$$
\mathscr{Y}(t)=\left\{\varrho \in \mathbb{R}:\left|v_{1}(t)-\varrho\right| \leq m(t)|x(t)-\bar{x}(t)|\right\} .
$$

As the multivalued operator $\mathcal{Y}(t) \cap F(t, \bar{x}(t))$ is measurable (Proposition III.4 in [30]), we can find a function $v_{2}(t)$ which is a measurable selection for $\mathscr{Y}$. So $v_{2}(t) \in F(t, \bar{x}(t))$, and for each $t \in[a, b]$, we have $\left|v_{1}(t)-v_{2}(t)\right| \leq m(t) \mid x(t)-$ $\bar{x}(t) \mid$.

For each $t \in[a, b]$, let us define

$$
\begin{aligned}
h_{2}(t)= & I^{\alpha_{1}+\alpha_{2}} v_{2}(t)-\lambda I^{\alpha_{2}} \bar{x}(t) \\
& +\frac{(t-a)^{\gamma_{1}+\alpha_{2}-1}}{\Lambda \Gamma\left(\gamma_{1}+\alpha_{2}\right)}\left[\theta I^{\alpha_{1}+\alpha_{2}} v_{2}(\eta)-\lambda \theta I^{\alpha_{2}} \bar{x}(\eta)\right. \\
& \left.-I^{\alpha_{1}+\alpha_{2}} v_{2}(b)+\lambda I^{\alpha_{2}} \bar{x}(b)\right] .
\end{aligned}
$$

Thus,

$$
\begin{aligned}
\left|h_{1}(t)-h_{2}(t)\right|= & I^{\alpha_{1}+\alpha_{2}}\left(\left|v_{1}(t)-v_{2}(t)\right|\right)+|\lambda| I^{\alpha_{2}}|x(t)-\bar{x}(t)| \\
& +\frac{(b-a)^{\gamma_{1}+\alpha_{2}-1}}{|\Lambda| \Gamma\left(\gamma_{1}+\alpha_{2}\right)}\left[|\theta| I^{\alpha_{1}+\alpha_{2}}\left(\left|v_{1}(\eta)-v_{2}(\eta)\right|\right)\right. \\
& +|\lambda||\theta| I^{\alpha_{2}}|x(\eta)-\bar{x}(\eta)| \\
& +I^{\alpha_{1}+\alpha_{2}}\left(\left|v_{1}(b)-v_{2}(b)\right|\right) \\
& \left.+|\lambda| I^{\alpha_{2}}|x(b)-\bar{x}(b)|\right] \\
\leq & \left\{\frac{(b-a)^{\alpha_{1}+\alpha_{2}}}{\Gamma\left(\alpha_{1}+\alpha_{2}+1\right)}+\frac{(b-a)^{\gamma_{1}+\alpha_{2}-1}}{|\Lambda| \Gamma\left(\gamma_{1}+\alpha_{2}\right)}\right. \\
& \left.\cdot\left[\frac{(b-a)^{\alpha_{1}+\alpha_{2}}}{\Gamma\left(\alpha_{1}+\alpha_{2}+1\right)}+|\theta| \frac{(\eta-a)^{\alpha_{1}+\alpha_{2}}}{\Gamma\left(\alpha_{1}+\alpha_{2}+1\right)}\right]\right\} \\
& \cdot\|m\|\|x-\bar{x}\|+\left\{|\lambda| \frac{(b-a)^{\alpha_{2}}}{\Gamma\left(\alpha_{2}+1\right)}\right. \\
& +\frac{(b-a)^{\gamma_{1}+\alpha_{2}-1}}{|\Lambda| \Gamma\left(\gamma_{1}+\alpha_{2}\right)}\left[|\lambda| \frac{(b-a)^{\alpha_{2}}}{\Gamma\left(\alpha_{2}+1\right)}\right. \\
& \left.\left.+|\lambda||\theta| \frac{(\eta-a)^{\alpha_{2}}}{\Gamma\left(\alpha_{2}+1\right)}\right]\right\}\|x-\bar{x}\|=\left(\|m\| \Omega_{1}\right. \\
& +\Omega(\|x-\bar{x}\| .
\end{aligned}
$$


Hence

$$
\left\|h_{1}-h_{2}\right\| \leq\left(\|m\| \Omega_{1}+\Omega_{2}\right)\|x-\bar{x}\|
$$

By interchanging the roles of $x$ and $\bar{x}$, one can obtain in a similar manner that

$$
H_{d}(\mathscr{F}(x), \mathscr{F}(\bar{x})) \leq\left(\|m\| \Omega_{1}+\Omega_{2}\right)\|x-\bar{x}\|,
$$

This shows that $\mathscr{F}$ is a contraction. So, by Covitz and Nadler's fixed-point theorem [29], operator $\mathscr{F}$ has a fixedpoint $x$ which corresponds to a solution of problem (3)-(4). This completes the proof.

Example 18. Consider the three-point boundary value problem of the Langevin inclusion with the Hilfer fractional derivative of the form:

$$
\left\{\begin{array}{l}
{ }^{H} D^{(3 / 7),(2 / 3)}\left({ }^{H} D^{(5 / 7),(1 / 4)}+\frac{1}{10}\right) x(t) \in F(t, x(t)), \quad t \in\left[\frac{3}{5}, \frac{9}{5}\right], \\
x\left(\frac{3}{5}\right)=0, \quad x\left(\frac{9}{5}\right)=\frac{3}{4} x\left(\frac{7}{5}\right) .
\end{array}\right.
$$

where

$$
F(t, x)=\left[\frac{1+\sin |x|}{(15+t)^{2}},\left(\frac{t+1}{41}\right)\left(\frac{1+2|x|}{1+|x|}\right)\right]
$$

Here, $\alpha_{1}=3 / 7, \alpha_{2}=5 / 7, \beta_{1}=2 / 3, \beta_{2}=1 / 4, \lambda=1 / 10, a=$ $3 / 5, b=9 / 5, \eta=7 / 5$, and $\theta=3 / 4$. We can find that $\gamma_{1}=17 /$ $21, \gamma_{2}=11 / 14, \Lambda \approx 0.4879734002, \Omega_{1} \approx 5.461283890$, and $\Omega_{2} \approx 0.6208844245$. It is clear that $F$ is measurable for all $x$ $\in \mathbb{R}$. Now, we see that

$H_{d}(F(t, x), F(t, y)) \leq\left(\frac{t+1}{41}\right)|x-y|, \quad x, y \in \mathbb{R}, \quad t \in\left[\frac{3}{5}, \frac{9}{5}\right]$.

By choosing $m(t)=(t+1) / 41$, we have $\|m\|=14 / 205$ and also we obtain $d(0, F(t, 0)) \leq m(t), t \in[3 / 5,9 / 5]$. Then we find that

$$
\|m\| \Omega_{1}+\Omega_{2} \approx 0.9938501536<1 .
$$

Hence, by using Theorem 17, we get that the boundary value problem (75) has at least one solution on $[3 / 5,9 / 5]$.

\section{Data Availability}

No data were used to support this study.

\section{Conflicts of Interest}

The authors declare that there are no conflicts of interest regarding the publication of this paper.

\section{Acknowledgments}

This research was funded by King Mongkut's University of Technology North Bangkok (Contract no. KMUTNB-61KNOW-031).

\section{References}

[1] A. A. Kilbas, H. M. Srivastava, and J. J. Trujillo, Theory and Applications of the Fractional Differential Equations, NorthHolland Mathematics Studies, 204, Elsevier, Amsterdam, Netherlands, 2006.

[2] R. L. Magin, Fractional Calculus in Bioengineering, Begell House Publishers, 2006.

[3] K. Diethelm, The Analysis of Fractional Differential Equations, Lecture Notes in Mathematics, Springer, New York, NY, USA, 2010.

[4] F. Mainardi, Fractional Calculus and Waves in Linear Viscoelasticy, World Scientific, Singapore, 2010.

[5] J. Klafter, S. C. Lim, and R. Metzler, Eds., Fractional Dynamics in Physics, World Scientific, Singapore, 2011.

[6] B. Ahmad, A. Alsaedi, S. K. Ntouyas, and J. Tariboon, Hadamard-Type Fractional Differential Equations, Inclusions and Inequalities, Springer, Cham, Switzerland, 2017.

[7] R. Hilfer, Ed., Applications of Fractional Calculus in Physics, World Scientific, Singapore, 2000.

[8] R. Hilfer, Y. Luchko, and Z. Tomovski, "Operational method for the solution of fractional differential equations with generalized Riemann-Liouvill fractional derivatives," Fractional Calculus \& Applied Analysis, vol. 12, pp. 299-318, 2009.

[9] R. Hilfer, "Experimental evidence for fractional time evolution in glass forming materials," Chemical Physics, vol. 284, no. 1-2, pp. 399-408, 2002.

[10] W. T. Coffey, Y. P. Kalmykov, and J. T. Waldron, The Langevin Equation, World Scientific, Singapore, 2nd edition, 2004.

[11] S. C. Lim, M. Li, and L. P. Teo, "Langevin equation with two fractional orders," Physics Letters A, vol. 372, no. 42, pp. 6309-6320, 2008.

[12] S. C. Lim and L. P. Teo, "The fractional oscillator process with two indices," Journal of Physics A: Mathematical and Theoretical, vol. 42, no. 6, p. 065208, 2009.

[13] M. Uranagase and T. Munakata, "Generalized Langevin equation revisited: mechanical random force and self-consistent structure," Journal of Physics A: Mathematical and Theoretical, vol. 43, no. 45, p. 455003, 2010.

[14] S. I. Denisov, H. Kantz, and P. Hänggi, "Langevin equation with super-heavy-tailed noise," Journal of Physics A: Mathematical and Theoretical, vol. 43, no. 28, p. 285004, 2010.

[15] A. Lozinski, R. G. Owens, and T. N. Phillips, "The Langevin and Fokker-Planck Equations in Polymer Rheology," Handbook of Numerical Analysis, vol. 16, pp. 211-303, 2011.

[16] L. Lizana, T. Ambjörnsson, A. Taloni, E. Barkai, and M. A. Lomholt, "Foundation of fractional Langevin equation: harmonization of a many-body problem," Physical Review E, vol. 81, no. 5, article 051118, 2010.

[17] W. Yukunthorn, S. K. Ntouyas, and J. Tariboon, "Nonlinear fractional Caputo-Langevin equation with nonlocal Riemann-Liouville fractional integral conditions," Advances in Difference Equations, vol. 2014, no. 1, 2014.

[18] W. Sudsutad, S. K. Ntouyas, and J. Tariboon, "Systems of fractional Langevin equations of Riemann-Liouville and 
Hadamard types," Advances in Difference Equations, vol. 2015, no. 1, 2015.

[19] J. Tariboon, S. K. Ntouyas, and C. Thaiprayoon, "Nonlinear Langevin equation of Hadamard-Caputo type fractional derivatives with nonlocal fractional integral conditions," Advances in Mathematical Physics, vol. 2014, Article ID 372749, 15 pages, 2014.

[20] K. M. Furati, N. D. Kassim, and N. Tatar, "Existence and uniqueness for a problem involving Hilfer fractional derivative," Computers \& Mathematics with Applications, vol. 64, no. 6, pp. 1616-1626, 2012.

[21] H. Gu and J. J. Trujillo, "Existence of mild solution for evolution equation with Hilfer fractional derivative," Applied Mathematics and Computation, vol. 257, pp. 344-354, 2015.

[22] J. Wang and Y. Zhang, "Nonlocal initial value problems for differential equations with Hilfer fractional derivative," Applied Mathematics and Computation, vol. 266, pp. 850859, 2015.

[23] S. Asawasamrit, A. Kijjathanakorn, S. K. Ntouyas, and J. Tariboon, "Nonlocal boundary value problems for Hilfer fractional differential equations," Bulletin of the Korean Mathematical Society, vol. 55, no. 6, pp. 1639-1657, 2018.

[24] S. Harikrishnan, K. Kanagarajan, and E. M. Elsayed, "Existence and stibility results for Langevin equations with Hilfer fractional derivative," Results in Fixed Point Theory and Applications, vol. 2018, article 20183, 10 pages, 2018.

[25] A. Granas and J. Dugundji, Fixed Point Theory, Springer-Verlag, New York, NY, USA, 2003.

[26] M. A. Krasnoselskii, "Two remarks on the method of successive approximations," Uspekhi Matematicheskikh Nauk, vol. 10, pp. 123-127, 1955.

[27] A. Lasota and Z. Opial, "An application of the Kakutani-Ky Fan theorem in the theory of ordinary differential equations," Bulletin de l'Académie Polonaise des Sciences Série des Sciences Mathématiques, Astronomiques et Physiques, vol. 13, pp. 781786, 1965.

[28] K. Deimling, Multivalued Differential Equations, Walter De Gruyter, Berlin-New York, 1992.

[29] H. Covitz and S. B. Nadler, "Multivalued contraction mappings in generalized metric spaces," Israel Journal of Mathematics, vol. 8, no. 1, pp. 5-11, 1970.

[30] C. Castaing and M. Valadier, Convex Analysis and Measurable Multifunctions, Lecture Notes in Mathematics 580, SpringerVerlag, Berlin-Heidelberg-New York, 1977. 